\title{
Charged Particles' Acceleration through Reconnecting Current Sheets in Solar Flares
}

\author{
C. Gontikakis ${ }^{1}$, C. Efthymiopoulos ${ }^{1}$, and A. Anastasiadis ${ }^{2}$ \\ 1 Research Center for Astronomy, Academy of Athens, Soranou Efessiou 4, \\ GR-11527, Athens, Greece \\ cgontik@academyofathens.gr, cefthim@academyofathens.gr \\ 2 National Observatory of Athens, Institute for Space Applications and Remote \\ Sensing, GR-15236, Penteli, Greece \\ anastasi@space.noa.gr
}

Summary. This study focuses on charged particles' acceleration in Reconnecting Current Sheets (RCS) taking place during solar flares. A Harris type topology was used to describe the electric and magnetic field of reconnecting current sheets. The use of a Hamiltonian formalism provided analytical laws describing the motion of the particles. Numerical simulations of a thermal distribution of particles interacting with single or multiple current sheets are presented. The final kinetic energy distributions after an interaction with a single current sheet present short energy ranges. When thermal particles interact with multiple current sheets a broad kinetic energy distribution is created. However, it is shown that there is an upper bound in the amount of kinetic energy that the particles can gain.

\section{Introduction}

The magnetic reconnection taking place in current sheets releases the energy stored in the magnetic field, in various forms, but primarily as the energy of charged particles accelerated, e.g., by DC electric fields in the reconnecting region. Several facts indicate that magnetic reconnection is responsible for the energy release during solar flares [6]. The study of particles' orbits inside a current sheet can provide important information on the physics of the acceleration process. Simple models of magnetic and electric fields inside current sheets gave the first estimation of the kinetic energy gain for the Earth magnetotail [5] and for solar flares [4]. In a series of papers ([1],[3], [2]) we studied the physics of particles acceleration using a Harris type reconnection topology. Several results were derived using a Hamiltonian formalism. 


\section{Analytical Formulation of the Problem}

The Harris type topology represents the inner part of a current sheet where dissipation of the magnetic energy takes place. The electric and magnetic field components are

$$
E=(0,0, E), \quad B=\left(-y / a, \xi_{\perp}, \xi_{\|}\right) B_{0} \quad \text { for }|y| \leq a .
$$

In (1), $\xi_{\perp}=B_{\perp} / B_{0}$ and $\xi_{\|}=B_{\|} / B_{0}$. We set $B_{0}=100$ Gauss as a reference value of the main magnetic field component. A particle is considered as being inside the current sheet when $|y| \leq a, a=1$. In that case the particle is subject to acceleration. The equations of motion are

$$
\ddot{x}=\xi_{\|} \dot{y}-\xi_{\perp} \dot{z}, \quad \ddot{y}=-\xi_{\|} \dot{x}-y \dot{z}, \quad \ddot{z}=\epsilon+\xi_{\perp} \dot{x}+y \dot{y},
$$

where, $\epsilon$ is a scaled value of the electric field. The equations of motions can be cast in a Hamiltonian form which, using the translation symmetry with respect to the $\mathrm{x}$-axis, is reduced in a 2 d.o.f. problem, namely

$$
H=\frac{1}{2} p_{y}^{2}+\frac{1}{2}\left(c_{4}+\frac{1}{2} y^{2}\right)^{2}+\frac{1}{2}\left(I_{2}-\xi_{\perp} z+\xi_{\|} y\right)^{2}-\epsilon z
$$

where $p_{y}$, and $c_{4}=\dot{z}-y^{2} / 2$ are the canonical momenta and $I_{2}=\dot{x}-\xi_{\|} y+\xi_{\perp} z$ is a second integral of motion [1].

\section{Numerical Results}

\section{Poincaré surfaces of section}

The Poincaré sections shown in Fig. 1 yield an overview of the types of orbits encountered in the phase space of the problem under study. The orbits are
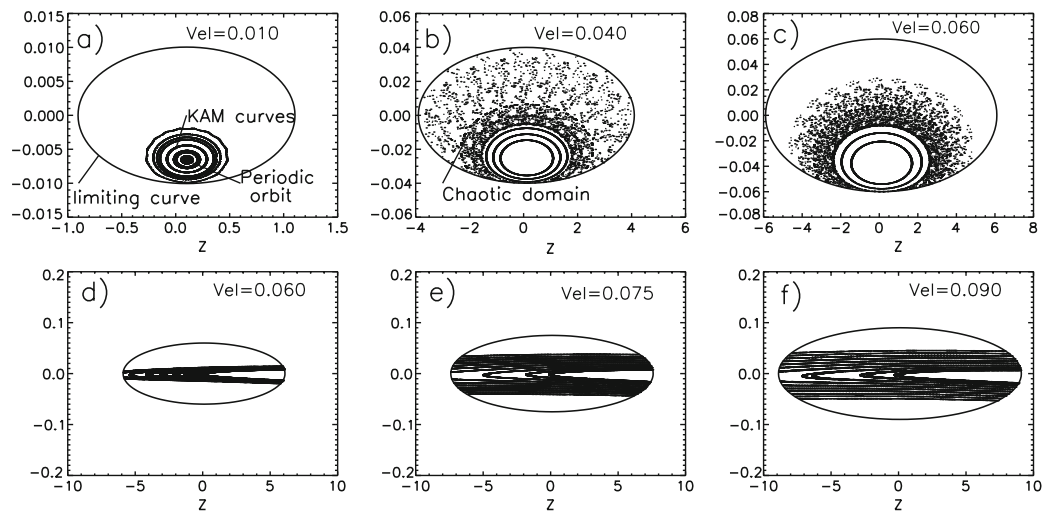

Fig. 1. Poincaré sections $(\dot{z}, z)$ for several initial velocities (indicated in the panels). For the first row: $\xi_{\perp}=10^{-2}, \xi_{\|}=0, \epsilon=10^{-5}$ and for the second row: $\xi_{\perp}=$ $10^{-2}, \xi_{\|}=1, \epsilon=10^{-5}$ 
traced on the plane $(\dot{z}, z)$ whenever they cross the $\mathrm{y}=0$ plane with a positive velocity. In all cases the particles' maximum velocity is restricted by a limiting curve derived by the condition of energy conservation. In the first row $\left(\xi_{\|}=0\right)$, the phase space shows KAM curves and a domain of chaotic motion. An adiabatic invariant exists on the KAM curves, in addition to the constant energy E and second integral $I_{2}$. In this case the regular orbits describe an oscillation of the particles as if the RCSs were acting like in a magnetic trap. When $\xi_{\|}=1$, the KAM curves (Fig. 1) are sections of ellipses, which dominate the phase space.

\section{Kinetic energy distribution of the accelerated particles}

We now present results from numerical simulations of the interaction of 30,000 particles, initially at thermal equilibrium $\left(10^{6} \mathrm{~K}\right.$, a typical coronal temperature), with a current sheet. Particles are injected from both sides and from the centre of the RCS $\left(y_{i n}=-1,0,1\right)$. Figure 2 shows the kinetic energy distributions for electrons (panels a,b) and protons (panels d,e) after such an interaction. The accelerated particles yield distributions in a very narrow

a) $\xi_{\|}=0 \quad \xi_{\perp}=1 . E-3 \quad \epsilon=1 . E-5$

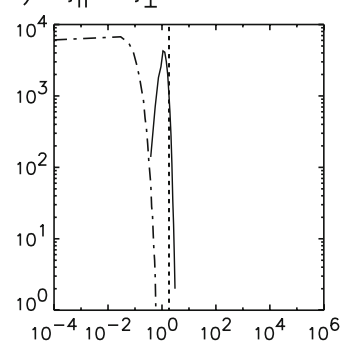

d) $\xi_{\| l}=0 \quad \xi_{\perp}=1 . E-3 \quad \epsilon=0.0184$

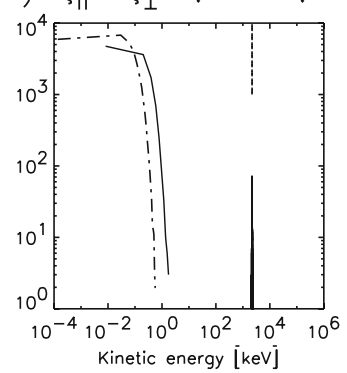

b) $\xi_{\|}=1 \quad \xi_{\perp}=1 . E-3 \quad \epsilon=1 . E-5$

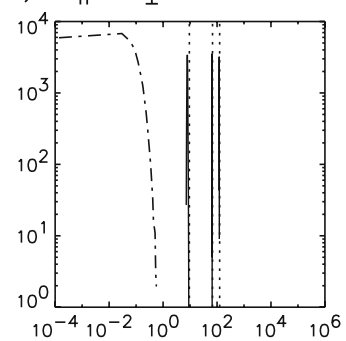

e) $\xi_{\| \mid}=1 \quad \xi_{1}=1 . E-3 \quad \epsilon=0.0184$

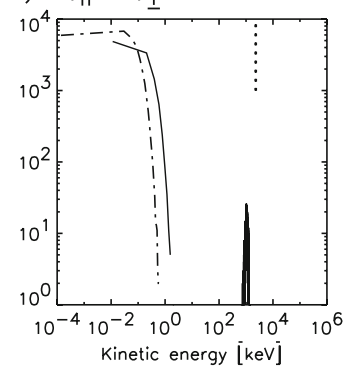

c)

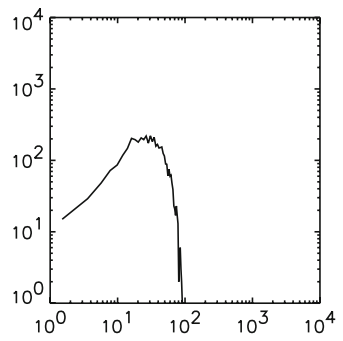

f)

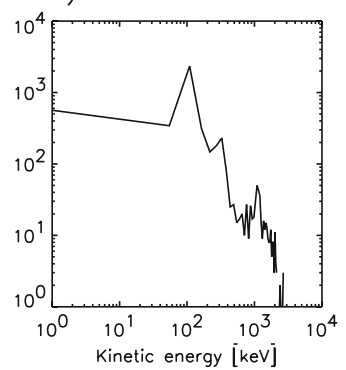

Fig. 2. Thermal distribution of particles interacting with single (panels a,b,d,e) or multiple (c,f) RCSs. The first row refers to electrons and the second row to protons. The RCS parameters for a single particle-RCS interaction are shown above each panel. The dot-dashed lines show the initial thermal distributions. The vertical lines show the analytic approximation (4). Sixty to seventy percent of protons are not accelerated by the current sheet 
range of energies. This can be explained as follows: Using the Hamiltonian (see $(3)$ ) with an initial kinetic energy $E_{0}$, one can find the following relation for the acceleration length along the Z-axis

$$
z_{\text {min }, \text { max }}=\frac{1}{\xi_{\perp}^{2}}\left(\xi_{\perp} I_{2}+\xi_{\|} \xi_{\perp} y_{\text {out }}+\epsilon \pm \sqrt{2 \xi_{\perp} I_{2} \epsilon+2 \xi_{\|} \xi_{\perp} y_{\text {out }} \epsilon+\epsilon^{2}+2 \xi_{\perp}^{2} E_{0}}\right)
$$

$\Delta E_{\min , \max }=\epsilon z_{\min , \max }$ are the constraints on the kinetic energy that a particle can gain. The three peaks when $\xi_{\|} \neq 0$ (Fig. 2b) correspond to the three different injection points along the y-axis (such peaks also exist in the case of Fig. 2e but they are hardly visible on the scale of the figure). The $z_{\min , \max }$ function depends on the injection points in (4) through $I_{2}\left(y_{0}\right)$. In our next numerical experiment, we studied the consecutive events of acceleration of particles with an initially thermal distribution interacting with 10 different current sheets. This experiment simulates the acceleration process in a solar flare where a large number of reconnecting current sheets are present. The parameters for each current sheet are selected randomly. The experiment shows (Fig. 2c,f) that the kinetic energy distribution is converging to a final form with maximum kinetic energy of $100 \mathrm{keV}$ (for electrons) and $1 \mathrm{MeV}$ (for protons). The final form of the distributions are obtained after six interactions. This result can be explained using (4). It can be shown ([2]) that as the kinetic energy of a particle becomes large, it is less probable that a current sheet can increase its kinetic energy by a substantial amount.

\section{Conclusions}

We studied the acceleration of particles through a current sheet using a Harris type configuration. A Hamiltonian treatment provided us with an analytical expression restricting the amount of kinetic energy that a particle can gain. For that reason thermal particles accelerated through a single RCS have narrow kinetic energy distributions. On the other hand, when accelerated through many consecutive RCSs, the particles acquire a final kinetic energy distribution reaching values up to a few $100 \mathrm{keV}$ for electrons or a few $\mathrm{MeV}$ for protons.

\section{References}

1. Efthymiopoulos C., Gontikakis C., Anastasiadis A., Astron. Astrophys., 443, 663 (2005)

2. Gontikakis C., Anastasiadis A., Efthymiopoulos C., Mon. Not. R. Astr. Soc., 378, 1019 (2007)

3. Gontikakis C., Efthymiopoulos C., Anastasiadis A., Mon. Not. R. Astr. Soc., 368, 293 (2006) 
4. Litvinenko Y.E., Astrophys. J., 462, 997 (1996)

5. Speiser T.W., J. Geophys. Res., 70, 4219 (1965)

6. Priest E., \& Forbes T., Magnetic Reconnection, 1st edn (Cambridge University Press, Cambridge, 2000) 